\title{
Behavior of Bulk Melt-Textured YBCO Single Domains Subjected to Crossed Magnetic Fields
}

\author{
Ph. Vanderbemden, S. Dorbolo, N. Hari-Babu, A. Ntatsis, D. A. Cardwell, and A. M. Campbell
}

\begin{abstract}
We have experimentally investigated the crossed magnetic field effects on bulk melt-processed YBCO single domains. The samples were first permanently magnetized along their $c$-axis and then subjected to several cycles of a transverse magnetic field parallel to the $a b$ planes. The magnetic properties along the $c$ and $a b$ directions were simultaneously measured using a couple of orthogonal pick-up coils as well as a Hall probe placed against the sample surface. The effects of both sweep amplitude and polarity were investigated. Field sweeps of alternate polarities are shown to affect the decay of the $c$-axis magnetization much more strongly than field sweeps of unique polarity do. However, the $c$-axis magnetization does not show any saturation even after a large number of field sweeps. Next, a micro-Hall probe scanning system was used to measure the distribution of magnetic induction over the top surface of the single domain subjected to the same combination of magnetic fields. The results are shown to be consistent with those determined with the sensing coils and bring out the role played by geometric effects.
\end{abstract}

Index Terms-Critical current, crossed fields, magnetic measurements, melt-textured (RE)BCO materials.

\section{INTRODUCTION}

$\mathbf{T}$ $\mathrm{HE}$ potential of bulk melt-processed $\mathrm{YBa}_{2} \mathrm{Cu}_{3} \mathrm{O}_{7}$ (YBCO) single domains to trap significant magnetic inductions at $77 \mathrm{~K}$ makes them particularly attractive for a variety of engineering applications including permanent magnets, magnetic bearings and brushless ac machines [1]-[4]. In many topologies to be envisaged for designing an efficient electrical motor based on superconductors, the bulk YBCO piece is permanently magnetized parallel to its $c$-axis and placed in the machine rotor [3]. In this configuration, the rotating magnetic field produced by the stator windings causes the superconducting single domain to experience an alternating magnetic field component parallel to its $a b$ planes and interacting with the $c$-axis magnetization. This corresponds thus to a situation where the material is subjected to crossed magnetic fields.

The problem of irreversible type-II superconductors subjected to crossed fields is quite intricate because the magnetic

Manuscript received August 5, 2002. This work was supported by the European Commission through the RTN 'SUPERMACHINES' contract (HPRN-CT-2000-0036) and by the Région Wallonne (RW) through the 'VESUVE' contract. Ph. Vanderbemden is recipient of an FNRS Post Doctoral Fellowship. Thanks to RW, FNRS and ULg for cryofluid and equipment grants. $\mathrm{Ph}$. Vanderbemden and S. Dorbolo are with the IRC in Superconductivity, University of Cambridge, U.K., for two months and are now with the SUPRAS research group, Department of Electrical Engineering and Computer Science (Montefiore Institute), University of Liège, Belgium (e-mail: Philippe.Vanderbemden@ulg.ac.be; S.Dorbolo@ulg.ac.be).

N. Hari-Babu, A. Ntatsis, D. A. Cardwell, and A. M. Campbell are with the IRC in Superconductivity, University of Cambridge, U.K. (e-mail: nhb24@cam.ac.uk; an249@cam.ac.uk; dc135@cam.ac.uk; amc1@cam.ac.uk).

Digital Object Identifier 10.1109/TASC.2003.812538 properties are controlled by flux-line pinning as well as flux-line cutting, i.e., the cross joining of nonparallel vortices at their intersection. In order to describe the experimental observations, several theoretical approaches were developed, among these the double critical-state model [5], the two-velocity hydrodynamic model [6] and numerical methods [7] involving a 3D extension of the Bean model [8].

It is now well established experimentally that the magnetization $M_{z}$ of a type-II superconducting sample placed in an external dc magnetic field $H_{z}$ is strongly decreased by applying a magnetic field $H_{y}$ perpendicular to both $M_{z}$ and $H_{z}$ [9], [10]. It has also been observed [11]-[14] that subsequent sweeps of this transverse field $H_{y}$ give rise to further reductions of $M_{z}$ down to some value corresponding to the limit case for which $H_{y}$ is an ac magnetic field applied orthogonal to the dc field $H_{z}$ [6], [15]. These phenomena have been observed even when the tilt of the total field is small $\left(H_{y} \ll H_{z}\right)$, but it should be emphasized that, in all cases, the magnitude of the transverse field $H_{y}$ is comparable to the penetration field parallel to $y$.

The aim of the present paper is to complement the set of experimental investigations mentioned above through carrying out specific crossed-field measurements on large bulk YBCO artifacts. The chosen magnetic field configuration, which no theory has attempted, corresponds to that used in electrical machines and for which the permanent magnetic moment $(\| c)$ and the transverse field $(\| a b)$ are directed respectively perpendicular and parallel to the largest face of the pellet. This geometry will also enable us to measure the distribution of the $c$-axis magnetic flux before and after applying the field sweeps in the $a b$ direction.

\section{EXPERIMENT}

Bulk melt-processed YBCO single domains were prepared by a top seeding technique described in [16]-[18]. The disk-shaped samples have a typical size of $2 \mathrm{~cm}$ diameter and $1 \mathrm{~cm}$ thickness.

The specimen was first permanently magnetized along the $c$-axis by field cooling down to $T=77 \mathrm{~K}$ under $0.5 \mathrm{~T}$. Then the field was switched off and the sample was rotated so that a series of magnetic field cycles could be applied in the $a b$ planes. All measurements were performed at $T=77 \mathrm{~K}$. Two orthogonal pick-up coils closely wound around the sample were used to measure simultaneously the magnetic properties along $c$ and $a b$. Both pick-up coil voltages were amplified, recorded and numerically integrated to get the average magnetic induction in each direction. In addition, an AREPOC Hall probe $(2 \mathrm{~mm} \times 3$ $\mathrm{mm}$ ) was stuck against the sample surface to measure the central induction parallel to the $c$-axis. 
The spatial distribution of the trapped magnetic induction was also measured by scanning a miniature Hall probe fixed to a motor-driven $x y z$ micro-positioning system over the top surface of the specimen parallel to both $x$ and $y$ with a step size of 1 $\mathrm{mm}$ in each direction. The distance between the Hall probe and the sample was $1 \mathrm{~mm}$, the active area of the Hall probe was 0.1 $\mathrm{mm} \times 0.1 \mathrm{~mm}$.

Prior to performing any measurement sequence, the sample position was carefully adjusted above $T_{c}$ in order to make sure the Hall probe signal was sensitive to the $c$-axis component of the local induction only and not to the field sweeps parallel to the $a b$ planes. Then the sample was firmly clamped to prevent the magnetic torque generated by the crossed fields from modifying this position.

A commercial Quantum Design Physical Property Measurement System (PPMS) was used to carry out additional AC and DC magnetic measurements on small pieces extracted from the single domain.

\section{RESULTS AND DISCUSSION}

\section{A. Sample Characterization}

Before performing the crossed-fields experiments, the magnetization loops $M(H)$ of the whole pellet were measured with the field applied in one definite direction. The full-penetration fields $\mu_{0} H_{p}$ determined for $H \| a b$ and $H \| c$ are $0.25 \mathrm{~T}$ and $0.32 \mathrm{~T}$ respectively. This latter value confirms that a field cooled (FC) procedure under $0.5 \mathrm{~T}$ (the maximum field which could be applied with the experimental system described above), is sufficient to generate the maximum remanent magnetization of our sample. The corresponding $c$-axis induction given by the Hall probe stuck at the sample center is $0.22 \mathrm{~T}$, which is smaller than the expected central value, i.e., $\mu_{0} H_{p} \| c=0.32 \mathrm{~T}$. This underlines the fact that the AREPOC Hall probe gives us the induction averaged over its own surface, and not the true central value.

After having performed the crossed-fields experiments, several cubic samples were extracted from the original disk-pellet and their magnetic properties were measured in order to investigate the homogeneity of the superconducting properties throughout the material. Both $T_{c}$ and $J_{c}$ were found to decrease going from the top to the bottom surface, whereas the homogeneity going from the center to the edge of the disk was quite satisfactory. This implies two consequences which are relevant to the crossed-fields measurements presented below: (i) at $T=$ $77 \mathrm{~K}$, the true superconducting part of the sample is "thinner" than the geometric thickness of the disk, and (ii) the field distribution measured over the top-surface of the disk reflects the intrinsic behavior of vortices and not an extrinsic behavior caused by macroscopic inhomogeneities.

\section{B. Measurements in the Crossed Fields Configuration}

Fig. 1 shows the influence of magnetic field sweeps parallel to the $a b$ planes on the average $c$-axis trapped induction $\langle B\rangle$ (measured by the sensing coil). The behavior of the $c$-axis induction in the central zone $B(r=0)$ (measured by the Hall probe) is plotted on the same graph. Both parameters are normalized with respect to their initial value. The experiment was repeated for different field amplitudes $H_{\max } \| a b$, and the remaining nor-

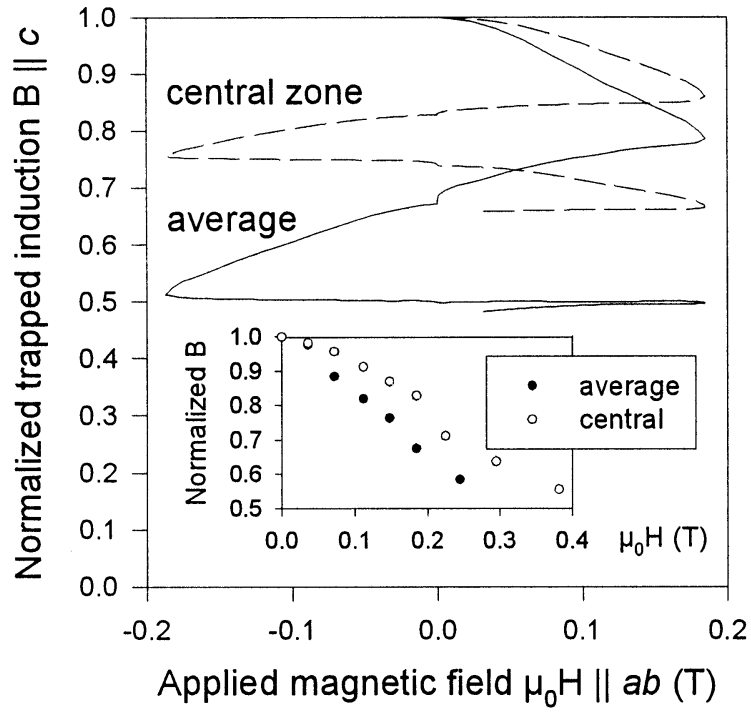

Fig. 1. Comparison of the average and the central induction $\| c$ during the application of a cycle of transverse field $\| a b$. The inductions are normalized with respect to their initial value. Inset: field amplitude dependence of the normalized induction after the application of the first sweep.

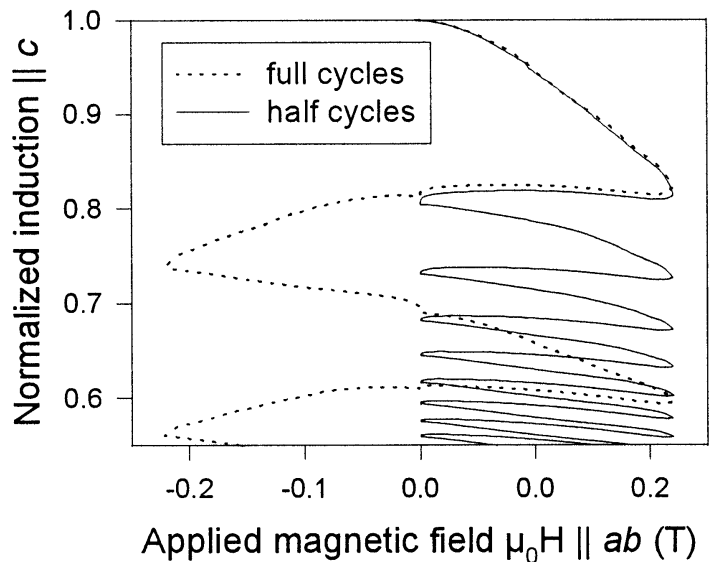

Fig. 2. Comparison of the decay of the central induction caused by full and half (positive) cycles of the transverse magnetic field.

malized inductions after each first sweep $0 \rightarrow H_{\max } \rightarrow 0$ are summarized in the inset of Fig. 1. As can be seen, the relative effects of a transverse field are always more marked for $\langle B\rangle$ than for $B(r=0)$. Qualitatively speaking, such a behavior supports the picture in which the transverse field may destroy the $a b$-plane currents flowing at the outer perimeter of the sample, since the effects of a suppression of $J_{c}$ flowing near the sample edges will always be stronger for the average induction than for the central value.

The influence of the polarity of field sweeps is shown in Fig. 2 which compares the effects of full cycles of $H \| a b$ (oscillating between $+H_{\max }$ and $-H_{\max }$ ) to those of half cycles ( $H$ oscillating between $+H_{\max }$ and 0 ). The alternate polarity sweeps are shown to affect the decay of the $c$-axis magnetization much strongly than sweeps of unique polarity do. In view of applications in machines, it is of interest to investigate the influence of a large number of such sweeps on the remanent magnetization. The results are presented in Fig. 3 which displays the normalized $c$-axis central induction at the end of each half-cycle. 


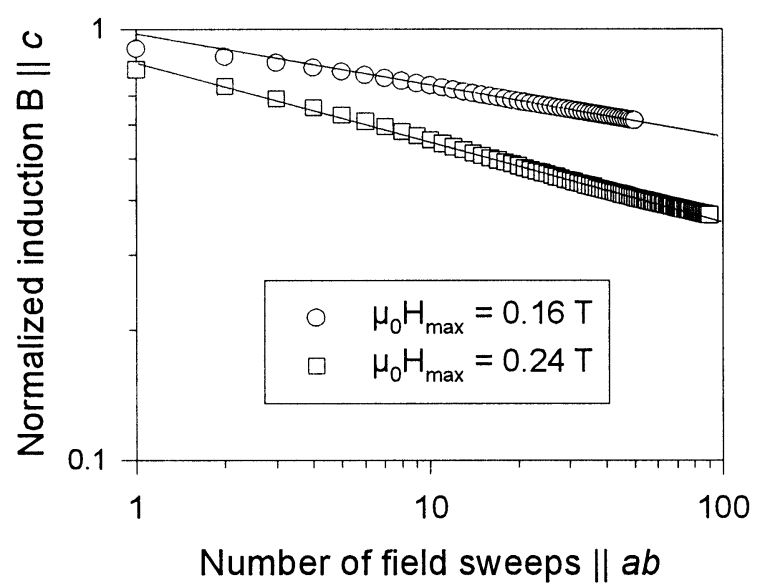

Fig. 3. Log-log plot of the central induction at the end of each half cycle for two different transverse field sweep amplitudes.

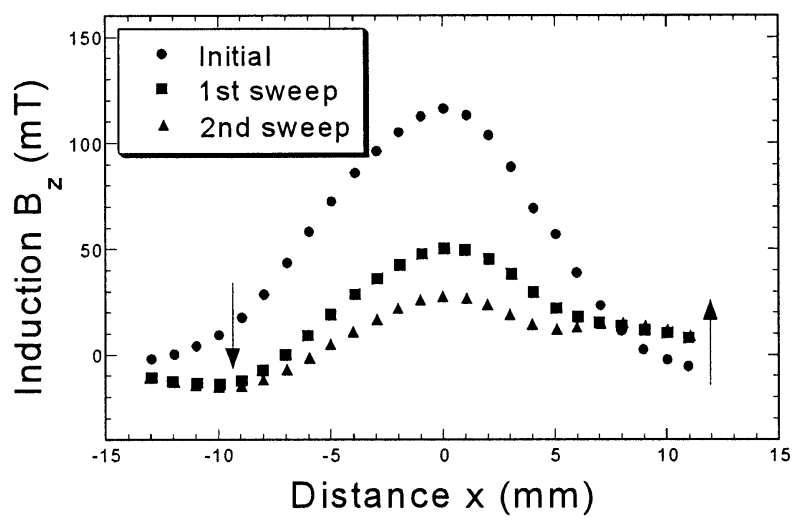

Fig. 4. Flux profiles measured along the transverse magnetic field direction.

Since each of these causes the induction to drop by smaller and smaller amounts, a log-log plot was used to analyze the data. The striking feature appearing in Fig. 3 is that the $c$-axis magnetization does not show any apparent saturation even after a large number of field sweeps. More precisely, the $c$-axis induction $B$ vs. cycle number $N$ curves can be fitted using a power law $B \sim N^{-n}$, with the exponent $n$ being directly related to the transverse field amplitude $H_{\max }$. In the future, it would be interesting to compare these results to those obtained by applying an orthogonal ac magnetic field (e.g., $50 \mathrm{~Hz}$ ) for a long time in order to estimate the number of field sweeps required to reach the saturation value mentioned in the literature [6], [15].

From the above experimental results, it can be concluded that the decay of the $c$-axis magnetization with $a b$-plane field is a complex process and that the junction between the transient study (i.e., the first sweeps) and the ac regime might involve a very large number of field sweeps.

\section{Hall Probe Mapping Results}

The influence of a transverse magnetic field on the trapped magnetic flux distribution has been illustrated by performing the following experiment. The sample was first magnetized along the $c$-axis and the trapped flux profile was recorded. Then a field $\mu_{0} H=0.5 \mathrm{~T} \| a b$ was applied along the $x>0$ direction, switched off and the flux mapping was carried out again. The same procedure was repeated a second time. Figs. 4 and 5 show

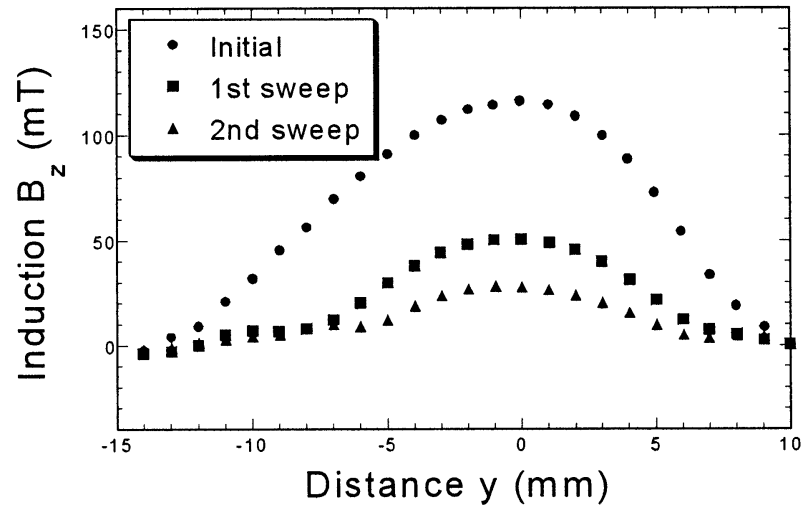

Fig. 5. Flux profiles measured along the direction perpendicular to the transverse magnetic field.

the distribution of the trapped induction along the directions respectively parallel $(x)$ and perpendicular $(y)$ to the field $H \| a b$. The initial profiles along both directions display a symmetric structure, thereby confirming the homogeneity of the single domain.

Owing to the cylindrical symmetry of the sample, one might expect this geometric effect not to affect the induction measurement in the $x=0$ plane. Therefore the genuine influence of the transverse field on the $c$-axis magnetization can be observed in the $B_{z}(x=0, y)$ plot. Indeed, the corresponding flux profiles (Fig. 5) are almost symmetrical with respect the $y=0$ axis. The field sweeps are seen to affect the flux profile in such a way they produce relatively 'flat' zones in the outer perimeter of the sample where the induction B nearly drops to 0 . This agrees with the results presented in Fig. 1, for which it was speculated that the transverse field affects the external shielding currents.

As expected, the transverse field reduces the $c$-axis induction but perceptible differences appear between the $x$ and $y$ directions. First we examine the field distribution along the $x$ axis (Fig. 4). The $B_{z}(x, y=0)$ plots are slightly tilted as indicated by the arrows in Fig. 4. This behavior can be understood qualitatively by considering demagnetization effects and by taking into account that the Hall probe is located at a finite distance from the sample top surface. After the first sweep, the sample is permanently magnetized along the $x$-axis. Since the induction lines are necessarily closed, they find a return path in the free space, in particular above the top-surface of the disk. This produces an additional $z$-component of the induction measured by the Hall probe. This component is positive for $x>0$ and negative for $x<0$, resulting in a distorted flux profile. Measurements with fields directed toward $x<0$ (not shown here) yield the opposite phenomenon. Therefore the behavior depicted in Fig. 4 is merely a geometric effect caused by the trapped induction in the transverse field direction.

\section{CONCLUSION}

In the present study, the properties of a bulk melt-textured YBCO single domain of large size have been measured in the crossed-field configuration using different techniques. The obtained results agree with each other and provide an experimental evidence that the application of a magnetic field orthogonal to the trapped field causes a suppression of the $a b$-plane currents 
flowing at the sample perimeter. It has also been found that the induction decay caused by a large number of transverse field sweeps follows a power law whose exponent is related to the sweep amplitude.

\section{ACKNOWLEDGMENT}

$\mathrm{Ph}$. Vanderbemden and A. Ntatsis would like to thank the European Commission for a travel grant. Tehy would also like to thank Profs. M. Ausloos, R. Cloots and H. W. Vanderschueren for many fruitful discussions.

\section{REFERENCES}

[1] A. M. Campbell and D. A. Cardwell, "Bulk high temperature superconductors for magnet applications," Cryogenics, vol. 37, pp. 567-575, 1997.

[2] S. I. Yoo, T. Higuchi, N. Sakai, H. Fujimoto, and M. Murakami, "RE-Ba-Cu-O for high functional superconducting permanent magnet," Mat. Sci. Engineering B, vol. 53, pp. 203-210, 1998.

[3] M. D. McCullogh and D. Dew-Hughes, "Brushless ac machines with high temperature superconducting rotors," Mat. Sci. Engineering B, vol. 53, pp. 211-215, 1998

[4] L. K. Kovalev et al., "Hysteresis electrical motors with bulk melt-textured YBCO," Mat. Sci. Engineering B, vol. 53, pp. 216-219, 1998.

[5] J. R. Clem and A. Pérez-González, "Flux-line-cutting and flux-pinning losses in type-II superconductors in rotating magnetic fields," Phys. Rev. $B$, vol. 30, pp. 5041-5047, 1984 .

[6] L. M. Fisher, A. V. Kalinov, S. E. Savel'ev, I. F. Voloshin, V. A. Yampol'skii, M. A. R. LeBlanc, and S. Hirscher, "Collapse of the magnetic moment in a hard superconductor under the action of a transverse ac magnetic field," Physica C, vol. 278, pp. 169-179, 1997.
[7] K. V. Bhagwat, S. V. Nair, and P. Chaddah, "Mutually perpendicular magnetic fields parallel to a slab: A test for the critical state model," Physica C, vol. 227, pp. 176-182, 1994.

[8] C. P. Bean, "Magnetization of high-field superconductors," Rev. Mod. Phys., vol. 1, pp. 31-36, 1964.

[9] S. J. Park and J. S. Kouvel, "Cross-flux effect as a vortex pinning process in grain-oriented $\mathrm{YBa}_{2} \mathrm{Cu}_{3} \mathrm{O}_{7}$, " Phys. Rev. B, vol. 48, pp 13 995-13 997, 1993.

[10] S. J. Park, J. S. Kouvel, H. B. Radousky, and J. Z. Liu, "Cross-flux effect as a vortex pinning process in $\mathrm{YBa}_{2} \mathrm{Cu}_{3} \mathrm{O}_{7}$ and $\mathrm{Y}_{0.8} \mathrm{Pr}_{0.2} \mathrm{Ba}_{2} \mathrm{Cu}_{3} \mathrm{O}_{7}$ crystals," Phys. Rev. B, vol. 48, pp. 13998-14000, 1993.

[11] L. M. Fisher, K. V. Il'enko, A. V. Kalinov, M. A. R. LeBlanc, F. Pérez-Rodríguez, S. E. Savel'ev, I. F. Voloshin, and V. A. Yampol'skii, "Suppression of the magnetic moment under the action of a transverse magnetic field in hard superconductors," Phys. Rev. B, vol. 61, pp. $15382-15391,2000$

[12] A. F. Caballo-Sánchez, F. Pérez-Rodríguez, and A. Pérez-González, "Magnetic response of hard superconductors subjected to parallel rotating magnetic fields," J. Appl. Phys., vol. 90, pp. 3455-3461, 2001.

[13] F. Pérez-Rodríguez, M. A. R. LeBlanc, and G. Gandolfini, "Flux-line cutting in granular high- $T_{c}$ and semi-reversible classical type-II superconductors," Supercond. Sci. Technol., vol. 14, pp. 386-397, 2001.

[14] M. A. R. LeBlanc, S. Celebi, and M. Rezeq, "Generation of quasireversibility in a commercial $\mathrm{Bi}: 2223 / \mathrm{Ag}$ tape by vortex shaking with varying orthogonal magnetic fields," Physica $C$, vol. 361, pp. 251-259, 2001.

[15] S. K. Hasanain, S. Manzoor, and A. Amirabadizadeh, "Magnetization and hysteresis of a melt-textured $\mathrm{YBa}_{2} \mathrm{Cu}_{3} \mathrm{O}_{7-x}$ in a crossed flux configuration," Supercond. Sci. Technol., vol. 8, pp. 519-524, 1995.

[16] D. A. Cardwell, "Processing and properties of large grain (RE)BCO," Mat. Sci. Engineering B, vol. 53, pp. 1-10, 1998.

[17] P. Vanderbemden, A. D. Bradley, R. A. Doyle, W. Lo, D. M. Astill, D. A. Cardwell, and A. M. Campbell, "Superconducting properties of natural and artificial grain boundaries in bulk melt-textured YBCO," Physica C, vol. 302, pp. 257-270, 1998 .

[18] P. Vanderbemden, R. Cloots, M. Ausloos, R. A. Doyle, A. D. Bradley, W. Lo, D. A. Cardwell, and A. M. Campbell, "Intragranular and intergranular superconducting properties of bulk melt-textured YBCO," IEEE Trans. Appl. Supercond., vol. 9, pp. 2308-23 311, 1999. 